\title{
Ion production and ionization effect in the atmosphere during the Bastille day GLE 59 due to high energy SEP particles
}

\author{
A.L. Mishev ${ }^{a, c, *}$ P.I.Y. Velinov ${ }^{b}$ \\ ${ }^{a}$ Space Climate Research Unit, University of Oulu, Finland \\ ${ }^{\mathrm{b}}$ Institute for Space Research and Technology, Bulgarian Academy of Sciences, Bl. \\ 1 Acad. G. Bonchev str. 1113 Sofia, Bulgaria \\ ${ }^{\mathrm{c}}$ Sodankylä Geophysical Observatory (Oulu unit), University of Oulu, Finland
}

\begin{abstract}
The influence of high energy particles, specifically cosmic rays, on atmospheric physics and chemistry is highly discussed. In most of the proposed models the role of ionization in the atmosphere due to cosmic rays is not negligible. Moreover, effect(s) on minor constituents and aerosols are recently observed, specifically over the polar regions during strong solar particle events. According to the recent findings for such effects it is necessary an essential increase of ion production, specifically during the winter period. The galactic cosmic rays are the main source of ionization in the Earth's stratosphere and troposphere. Occasionally, the atmospheric ionization is significantly enhanced during strong solar energetic particles events, specifically over the polar caps. During the solar cycle 23 several strong ground level enhancements were observed. One of the strongest was the Bastille day event occurred on 14 July 2000. Using a full Monte Carlo 3-D model, we compute the atmospheric ionization, considering explicitly the contribution of cosmic rays with galactic and solar origin, focusing on high energy particles. The model is based on atmospheric cascade simulation with the PLANETOCOSMICS code. The ion production rate is computed as a function of the altitude above the sea level. The ion production rate is computed on a step ranging from 10 to 30 minutes throughout the event, considering explicitly the spectral and angular characteristics of the high energy part of solar protons as well as their time evolution. The corresponding event averaged ionization effect relative to the average due to galactic cosmic rays is computed in lower stratosphere and upper troposphere at various altitudes, namely $20 \mathrm{~km}, 15 \mathrm{~km}, 12 \mathrm{~km}$ and $8 \mathrm{~km}$ above the sea level in a sub-polar and polar regions. The $24^{h}$ and the weekly ionization effects are also computed in the troposphere and low stratosphere. Several applications are discussed.
\end{abstract}

Key words: Atmospheric ionization, Cosmic rays, Solar energetic particles, 


\section{Introduction}

The effect of high energy particles, specifically cosmic rays, on different atmospheric processes related to atmospheric chemistry and physics is extensively discussed (e.g. Bazilevskaya et al., 2008; Mironova et al., 2015, and references therein). In most of the recent models, the induced by energetic particles atmospheric ionization plays an important role. The induced by energetic particles atmospheric ionization affect atmospheric processes, global electric circuit and minor constituents in the Earth's atmosphere (e.g. Krivolutsky et al., 2006; Randall et al., 2007; Jackman et al., 2008; Rozanov et al., 2012; Nicoll and Harrison, 2014; Verronen et al., 2015). The populations of energetic particles inducing atmospheric ionization, include galactic cosmic rays (GCRs), solar energetic particles (SEPs), precipitating protons and electrons from radiation belts (e.g. Bazilevskaya et al., 2008; Dorman, 2009; Mironova et al., 2015, and references therein). While the solar UV and X-rays dominate at altitudes above some $100 \mathrm{~km}$ above the sea level (a.s.l.), but absorbed below, the most energetic particles are the main source of ionization below $100 \mathrm{~km}$ a.s.l. In this work we focus on high and very high energy particles with cosmic origin, namely GCRs and the high energy part of about $300 \mathrm{MeV} /$ nucleon and above SEPs, while the other populations are considered elsewhere (e.g. Mironova et al., 2015, and references therein).

The most important source of high energy particles inducing ionization in the troposphere and stratosphere of the Earth originate from outer space viz. cosmic rays (e.g. O'Brien, 1970; Dorman, 2004; Usoskin et al., 2009; Velinov et al., 2013). The majority of those particles are protons and $\alpha-$ particles, and minor quantities of heavier nuclei are also observed (e.g. Gaisser and Stanev, 2010, and references therein). Most of CR particles originate from the Galaxy, known as GCRs (e.g. Gaisser and Stanev, 2010, and references therein). The cosmic ray particles penetrate deep into the atmosphere, induce a complicated nuclear-electromagnetic-muon cascade, which eventually leads to an ionization of the ambient air (e.g. Dorman, 2004; Usoskin et al., 2009). The maximum of ion production in the atmosphere, observed at the altitude of about 12-

\footnotetext{
* Corresponding author:A.Mishev, Space Climate Research Unit, University of Oulu, Finland; Tel:(+358)44 9160753

Email address: alexander.mishev@oulu.fi, alex_mishev@yahoo.com (A.L. Mishev).
} 
$15 \mathrm{~km}$ a.s.l., is known as Pfotzer-Regener maximum (Regener and Pfotzer, 1935; Pfotzer, 1936). The CR flux is modulated in the Heliosphere, follows the inverse 11-year solar cycle and also responds to transient phenomena e.g. Forbush decreases (Forbush, 1937).

A sporadic source of high energy particles penetrating the atmosphere and eventually inducing ionization follows eruptive solar processes on the Sun as solar flares and coronal mass ejections (CMEs), namely the so-called solar energetic particles (e.g. Reames, 1999; Cliver et al., 2004). The energy of SEPs is usually of the order of tens of $\mathrm{MeV} /$ nucleon. However, in some cases it can reach $\mathrm{GeV} /$ nucleon, leading to an atmospheric cascade and an enhancement of the count rate of ground based detectors, specifically neutron monitors (NMs). This special class of SEP events is called a ground level enhancement (GLE) (e.g. Shea and Smart, 1982; Dorman, 2004; Aschwanden, 2012). Their occurrence rate is roughly once per year, with increasing probability during maximum and decline phase of the solar cycle (Shea and Smart, 1990; Stoker, 1995; Bazilevskaya, 2005). These events usually lead to a significant increase of ion production in the atmosphere, specifically in polar and sub-polar region in the Earth's upper atmosphere,where the magnetospheric shielding is not as effective as at middle and equatorial latitudes (Jackman et al., 2011; Mishev et al., 2011; Usoskin et al., 2011b; Mironova et al., 2012; Mishev et al., 2013). Detailed study of ion production, accordingly ionization effect, which represents the ion production integrated over the event or a corresponding time interval relative to the ion production due to GCR computed prior to the event.

The ion production is computed in different parts of the Earth's atmosphere during GLEs. This allows one to estimate the possible effect of CR on atmospheric physics and chemistry in enhanced mode. The ion production rate during GLEs is determined mainly on SEP spectra and anisotropy and depends also on duration and apparent source location. Since, the GLE events differ from each other in spectra, duration, location occurrence, geomagnetic conditions as well as time evolution of the features related to the ion production (Gopalswamy et al., 2012; Moraal and McCracken, 2012), it is necessary to study each strong event separately. In this connection, here we study the ion production and the corresponding ionization effect during one of the strongest GLEs of solar cycle 23, namely the Bastille day GLE 59 on 14 July 2000. In this study we focus on a low stratosphere and upper troposphere, where only the most energetic part of SEPs play an important role, while the low energy part with important contribution at altitudes of $40 \mathrm{~km}$ above the level (e.g. Mironova et al., 2015) is not considered. 


\section{Model for cosmic ray induced ionization}

The ion production in the atmosphere induced by high energy particles can be assessed by analytical and/or parametrization models (e.g. O'Brien, 1970; Vitt and Jackman, 1996). However, the parametrization models usually suffer from lack of precision, specifically in the low stratosphere and troposphere and possess constrains to a given atmospheric region and/or cascade component (e.g. Velinov et al., 2013, and references therein). On the other hand full target models based on a full Monte Carlo simulation of the atmospheric cascade allow one to compute the ion production rate, accordingly ionization effect in the atmosphere considering all the physics process involved (Desorgher et al., 2005; Usoskin and Kovaltsov, 2006; Velinov et al., 2009). Therefore, the full target models permit a realistic computation of ion production rate, accordingly ionization effect in the atmosphere during major GLEs, explicitly considering the contribution of cosmic rays with galactic and/or solar origin (Mishev et al., 2011; Usoskin et al., 2011b; Mishev and Velinov, 2015).

In this work we employ a model similar to Usoskin and Kovaltsov (2006). The full description of the model is given elsewhere (Mishev and Velinov, 2007; Velinov et al., 2009). The ion production rate as a function of the altitude a.s.l. is:

$$
q(h, E)=\frac{1}{E_{\text {ion }}} \sum_{i} \int_{E_{\text {cut }}\left(R_{c}\right)}^{\infty} D_{i}(E) \frac{\partial E(h, E)}{\partial h} \rho(h) d E
$$

where $\partial E$ is the deposited energy in an atmospheric layer $\partial h, h$ is the air overburden (air mass) above a given altitude in the atmosphere expressed in $\mathrm{g} / \mathrm{cm}^{2}$ subsequently converted to altitude a.s.l., $D_{i}(E)$ is the differential cosmic ray spectrum for a given component $i$ : protons $\mathrm{p}$, Helium ( $\alpha$-particles), the latter also representative for heavier nuclei with atomic number $\mathrm{Z}>2$ similarly to Usoskin and Kovaltsov (2006); Mishev and Velinov (2011a,b), $\rho$

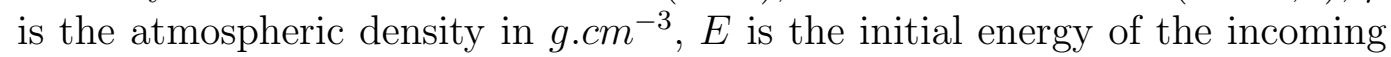
primary nuclei on the top of the atmosphere and $E_{i o n}=35 \mathrm{eV}$ is the average energy necessary for creation of an ion pair in air (Porter et al., 1976). In the case of SEPs, the $D_{i}(E)$ can be considered from ground based NM data analysis (e.g. Mishev and Velinov, 2015) or similarly to Usoskin et al. (2011b).

In Eq. (1) the integration is over the kinetic energy above $E_{c u t}\left(R_{c}\right)$, which is defined by the local cut-off rigidity $R_{c}$ for a nuclei of type $i$ at a given geographic location by the expression:

$$
E_{c u t, i}=\sqrt{\left(\frac{Z_{i}}{A_{i}}\right)^{2} R_{c}^{2}+E_{0}^{2}}-E_{0}
$$


where $E_{0}=0.938 \mathrm{GeV} / \mathrm{n}$ is the proton's rest mass.

For the computation of ion production rate due to GCR we assume the force field model according to Gleeson and Axford (1968); Burger et al. (2000) with parametrization of local interstellar spectrum according to Usoskin and Kovaltsov (2006). The modulation potential is computed similarly to Usoskin et al. (2011a). The SEPs spectra in equation (1), which considerably vary from event to event as well as throughout the event(s), are retrieved form NM data analysis. Note that the global NM network is sensitive to SEPs with rigidity greater than $1 \mathrm{GV}$ (the atmospheric cut-off) or $\approx$ $430 \mathrm{MeV} /$ nucleon. However, the high altitude polar NMs e.g. the South Pole allows one to scale the derived SEP spectra to about $300 \mathrm{MeV} /$ nucleon as considered in this study. The time evolution of SEP spectral and angular distribution throughout the event is explicitly considered according to (Bombardieri et al., 2006; Mishev and Usoskin, 2016). Here, we consider the high energy part of the GLE particles, namely above some $300 \mathrm{MeV} /$ nucleon, which plays an important role in tropospheric ionization, whcih is consistent with recent studies (Usoskin et al., 2009, 2011b).

The atmospheric cascade simulations are performed with the GEANT 4 (Agostinelli et al., 2003) based PLANETOCOSMICS code (Desorgher et al., 2005) assuming a realistic atmospheric model NRLMSISE 00 and considering explicitly the seasonal influence (Picone et al., 2002; Mishev and Velinov, 2010, 2014).

3 Ion production rate during Bastille day GLE 59 event

During the solar cycle 23, sixteen GLE events were observed (Gopalswamy et al., 2012). The observed increases varied between $3 \%$ and $269 \%$. The first event occurred on 6 November 1997 (GLE 55) and the last event occurred on 13 December 2006 (GLE 70). All those events differ in spectra and anisotropy, apparent source position as well as the time evolution of all features and characteristics, which determine the ion production (Moraal and McCracken, 2012).

The period of mid July 2000 was characterized by intense solar activity, resulting on three X-class solar flares and two halo coronal mass ejections (CME) (Dryer et al., 2001; Klein et al., 2001). The event was related to X5.8/3B solar flare and the associated full halo CME. The GLE onset began between 10:30 and 10:35 UT with strongest NM increases observed at South Pole $(58.3 \%)$ and SANAE (54.4\%) NMs. The event was characterized by a strong anisotropy in its initial phase and relatively hard spectra during the 
event onset (Bieber et al., 2002; Bombardieri et al., 2006; Mishev and Usoskin, 2016).

During major GLE events the ion production rate in the atmosphere is a superposition of the contribution due to GCRs, which are occasionally reduced (Forbush decrease) and SEPs, the latter typically possess an essential anisotropic part, specifically during the event onset and initial phase. The propagation of both GCRs and SEPs is affected by the geomagnetic field (shielding), which is most effective near to geomagnetic equator and negligible near to geomagnetic poles. The shielding is quantified by the effective vertical cut-off rigidity $R_{c}$, which varies with the geographical location (Cooke et al., 1991). In this study we compute the ion production rate and the corresponding ionization effect in the atmosphere in the sub-polar and polar regions of the Earth, where the expected effect is maximal, namely in regions with $R_{c} \approx 1 \mathrm{GV}$ (Mishev and Velinov, 2013, 2015). The anisotropy of SEPs is considered explicitly by computation of asymptotic cones in the region of interest similarly to Mishev and Velinov (2015). Note that, the asymptotic cone computations are explicitly function of the epoch and the geomagnetic conditions, therefore they vary from event to event (Kudela and Usoskin, 2004; Mishev and Usoskin, 2013). Herein, we consider the high energy part (above $300 \mathrm{MeV} /$ nucleon) of the GLE particles, which predominantly play role in the ion production in the stratosphere and troposphere, while the contribution of low energy part dominating at altitudes of about $40 \mathrm{~km}$ a.s.l. is planned for forthcoming work.

The computation of the cut-off rigidity and asymptotic cones of the region of interest, namely grid of $5^{\circ} \times 5^{\circ}$, is performed by realistic modelling of particle propagation in the magnetosphere similarly to McCracken et al. (1962); Shea et al. (1965); Smart et al. (2000); Desorgher et al. (2009); Mishev and Usoskin (2013). The particle propagation in the magnetosphere was modelled with the MAGNETOCOSMICS code (Desorgher et al., 2005) using the IGRF geomagnetic model as internal field (Langel, 1987) and Tsyganenko 1989 model as external field (Tsyganenko, 1989). This combination of models provides balance between simplicity and realism (Kudela and Usoskin, 2004; Kudela et al., 2008; Nevalainen et al., 2013). Since the event occurred during the recovery phase of a Forbush decrease, we explicitly consider the reduced GCR flux adjusted from NMs measurements, data retrieved from the neutron monitor database (www. NMDB.eu) (Mavromichalaki et al., 2011). The SEP spectral and angular characteristics are derived on the basis of NM measurements and subsequent analysis, the details are given elsewhere (Bombardieri et al., 2006; Mishev and Usoskin, 2016). The SEP spectral and angular characteristics are shown in Fig.1, the details are given in Table 1. 
For the GLE particles a modified power law rigidity spectrum with a variable slope is assumed similarly to Mishev and Usoskin (2016):

$$
J_{\|}(R)=J_{0} R^{-(\gamma+\delta \gamma(R-1))}
$$

where $J_{\|}(R)$ is the particle flux with given rigidity $R$ arriving from the Sun along the axis of symmetry whose direction is defined by geographic coordinate angles $\Psi$ and $\Lambda$ (latitude and longitude), $\gamma$ is the power-law spectral exponent at rigidity $\mathrm{R}=1 \mathrm{GV}, \delta \gamma$ is the rate of the spectrum steepening. Accordingly the pitch angle distribution is assumed to be a Gaussian:

$$
G(\alpha) \sim \exp \left(-\alpha^{2} / \sigma^{2}\right)
$$

where $\alpha$ is the pitch angle, $\sigma$ is parameter corresponding to the width of the pitch angle distribution.

The time evolution of the GLE rigidity spectrum, PAD and apparent source position is considered explicitly throughout the event on a step of 10, 15 and 30 min. during the initial and main phase of the event, accordingly $1 \mathrm{~h}$ during the late phase of the event. The SEP rigidity spectrum was gradually softening throughout the event, while the anisotropy was strong during the event's onset, but rapidly dropped resulting in a wide solar proton angular distribution (Bombardieri et al., 2006; Mishev and Usoskin, 2016). As a result, after the initial phase of the event, namely after 11:00 UT the ionization effect broaden out (see Section 4).

The ion production rates (Eq.1) for several periods throughout GLE 59 are presented in Fig. 2. In Fig. 2a are shown the ion production rates in the region with $R_{c} \approx 1 \mathrm{GV}$, accordingly in Fig. $2 \mathrm{~b}$ in the region with $R_{c} \approx 2 \mathrm{GV}$. The ion production rates due to SEPs in the region with $R_{c} \approx 1 \mathrm{GV}$ are greater than the average due to GCRs, but event onset when a considerable steepening of the SEP spectrum was observed (Table 1 ). In the region of $R_{c} \approx 2 \mathrm{GV}$ the ion production rates are less than the average due to GCRs, because of rapidly falling and steep SEP spectrum (e.g. Mishev and Usoskin, 2016).

The ion production rate profiles at 11:00 UT and 12:00 UT are comparable, because the very similar SEP spectra. In the late phase of the event the ion production rate diminishes, because the gradually softening of SEP spectra and decrease of GLE particles flux. In the region with $R_{c}$ $\geq 2$ the ion production rate diminishes constantly throughout the event, because the majority of GLE particles are shielded by the 
geomagnetic field, leading to a reduction of their flux. The ion production rate, accordingly the ionization effect (Section 4) is considerably affected by the SEPs anisotropy, specifically during the event initial phase. Since the apparent source position of SEPs is located close to the equatorial region accompanied by a fast isotropisation of the incoming SEP flux (Bombardieri et al., 2006; Mishev and Usoskin, 2016)), the ionization effect relative to the average due to GCR is more symmetric compared to the GLE 70 (Mishev and Velinov, 2015, 2016).

4 Relative to GCR ionization effect in the polar and sub-polar region

Here, we present computation of ionization effect relative to the average ionization due to GCR at various time scales, namely during the event, at $24^{h}$ and weekly scale, all important to estimate the possible SEP influence on atmospheric chemistry and physics (e.g. Bazilevskaya et al., 2008; Usoskin et al., 2009; Mironova et al., 2015, and references therein). Since we consider the high energy GLE particles, the ion production and ionization effect are computed for period corresponding to the observed NM increases retrieved from NMDB i.e. from the event onset to 00:00 UT of 15 July 2000 for the event averaged ionization effect, accordingly $24 \mathrm{~h}$ and $168 \mathrm{~h}$ after the event onset for the daily, respectively weekly ionization effect.

The event averaged ionization effect is computed as integration over the ion production throughout the event [ion pairs $\mathrm{cm}^{-3}$ ] (integration of ion production rate over the phase of the event applying a recombination model similarly to Krivolutsky et al. (2006)) relative to the production due to GCR, computed at the period prior to the event, but not affected by a Forbush decrease. The event averaged ionization effect in the polar and sub-polar region at altitude of 20 $\mathrm{km}$ a.s.l. is presented in Fig.3a and at altitude of $15 \mathrm{~km}$ a.s.l. in Fig.3b.

The ionization effect depends strongly on the altitude a.s.l. and geographic location. Since, SEP spectra are considerably softer than GCR, the maximum ionization effect is observed at higher altitudes than the produced by the average GCR. The maximum ionization effect at altitude of $20 \mathrm{~km}$ a.s.l. is roughly $90 \%$ located in the region of $30^{\circ} \mathrm{W}-30^{\circ} \mathrm{E}$ in a sub-polar and polar region in Northern hemisphere. The ionization effect in Northern hemisphere diminishes slightly in the region of $170^{\circ} \mathrm{W}-170^{\circ} \mathrm{E}$ to $\approx 65 \%$. The minimum ionization effect at altitude $20 \mathrm{~km}$ a.s.l. is observed in the Southern hemisphere in the region of 
$160^{\circ} \mathrm{W}-160^{\circ} \mathrm{E}$, where it is $\approx 50 \%$. The event averaged ionization effect is slightly greater at altitude of $15 \mathrm{~km}$ a.s.l. (Fig.3b), where it is roughly $95 \%$. The maximum ionization effect at altitude of $15 \mathrm{~km}$ a.s.l. is observed in the region of $30^{\circ} \mathrm{W}-30^{\circ} \mathrm{E}$ in the North sub-polar and polar region. Accordingly, the ionization effect at altitude of $15 \mathrm{~km}$ a.s.l. diminishes in the region of $160^{\circ} \mathrm{W}-160^{\circ} \mathrm{E}$ to $\approx 70 \%$ in Northern hemisphere, accordingly in Southern hemisphere to $\approx 55 \%$.

At lower altitudes the event averaged ionization effect gradually diminishes. At altitude of $12 \mathrm{~km}$ a.s.l. (Fig. $4 \mathrm{a}$ ) it is $\approx 85 \%$, specifically located in the region of $30^{\circ} \mathrm{W}-30^{\circ} \mathrm{E}$ in a sub-polar and polar region in the Northern hemisphere, where it is maximal. The ionization effect in Northern hemisphere diminishes in the region of $170^{\circ} \mathrm{W}-170^{\circ} \mathrm{E}$ to roughly $60 \%$. In the troposphere, at altitude of $8 \mathrm{~km}$ a.s.l., the ionization effect diminishes considerably to $\approx 40 \%$, the maximum observed in the region of $20^{\circ} \mathrm{W}-20^{\circ} \mathrm{E}$ (Fig.4b), accordingly the minimum of $\approx 30 \%$ is observed in the region $165^{\circ} \mathrm{W}-165^{\circ} \mathrm{E}$.

The $24^{h}$ averaged ionization effect is computed as integration over the ion production during the GLE taking into account solely the GCR contribution after the event (after 00:00 UT of 15 July), explicitly considering its flux variation according to NM data. It is compared with the ion production over $24^{h}$ produced by GCR computed at the period prior to the event, but not affected by a Forbush decrease. The $24^{h}$ averaged relative to GCR tropospheric ionization effect is computed in order to estimate the possible impact of SEPs on the Earth's atmosphere (e.g. Krivolutsky and Repnev, 2012). It is mainly function of the altitude a.s.l. and the accompanying Forbush decrease, which reduces the GCR flux. The $24^{h}$ ionization effect is uniformly distributed over the polar region. Its is in the order of $3-$ $4.6 \%$ at altitude of $12 \mathrm{~km}$ a.s.l. (Fig.5a) and $2.7-3.6 \%$ (Fig.5b) at altitude of $8 \mathrm{~km}$ a.s.l. Therefore, the ionization effect during GLE 59 on $24^{h}$ time scale due to the high energy part of SEP particles is minor in the troposphere and low stratosphere.

Similarly is computed the weekly ionization effect (Fig.6). It is computed as integration over the ion production during the GLE and considering the GCR after the event. The weekly averaged ionization effect relative to GCR is a function on the altitude a.s.l. and strongly depends on the accompanying Forbush decrease. The weekly averaged ionization effect is uniformly distributed over the polar region. It is clearly negative, in the order of $-1.8 \%$ at altitude of $12 \mathrm{~km}$ a.s.l. (Fig.6a) and $\approx-3.5 \%$ at altitude of $8 \mathrm{~km}$ a.s.l. (Fig.6b). Therefore, the ionization effect in the troposphere and low stratosphere due to high energy GLE particles on weekly time scale is mainly due 
to GCR flux, which in case of a Forbush decrease lead to slightly negative values, in accordance with (Usoskin et al., 2011b).

\section{Summary and Discussion}

The sporadic rapid change of CR flux, namely Forbush decreases and/or GLEs allows one to study the possible effects of high energy particles on atmospheric chemistry and physics on enhanced magnitude (e.g. Bazilevskaya et al., 2008; $\mathrm{Yu}$ and Luo, 2014; Mironova et al., 2015). At recent it was shown that for observation of such effects it is necessary an essential increase of ion production in the atmosphere, which can take place during strong GLEs, specifically during winter in order to avoid the eventual influence of UV (Mironova and Usoskin, 2014). Therefore, strong events as 20 January 2005 (Mishev et al., 2011, 2013), 13 December 2006 (Mishev and Velinov, 2013; Žigman et al., 2014; Mishev and Velinov, 2015) and the considered here Bastille day GLE 59 on 14 July 2000 are convenient for such a study.

In this work we computed the ion production rate and the corresponding ionization effect at various time scales, namely during the event, $24^{h}$ and weekly time scale, considering explicitly SEPs characteristics as well as their temporal evolution throughout the event. Here, we focus on ionization effect in the low stratosphere and upper stratosphere, specifically due to high energy part of GLE particles. One can see that the ion production rate is significant during the main phase of the event, specifically at the polar and sub-polar region. During the initial and late phases of the event the ion production is comparable to the average due to GCR. Moreover, at altitudes of about $10 \mathrm{~km}$ a.s.l. and below the ion production due to GCR is greater than the high energy part of SEPs, because of the rapidly falling spectra of the latter. At high mid latitudes and mid latitudes with cut-off rigidity $R_{c} \approx 2-3 \mathrm{GV}$, the ion production due to GCR dominates in the whole atmosphere and the corresponding ionization effect is marginal throughout the event.

The ion production vary throughout the event, mainly due to variation of spectral and angular characteristics of SEPs as well as the variation of apparent source position location. However, because of the fast isotropisation of SEPs, the role of the latter (slight movement to a South-West direction) is not as important as compared to GLE 70, but during the event onset and its initial phase (Mishev and Velinov, 2015). As a result the corresponding ionization effect is almost symmetrically distributed over both hemispheres, being maximal in the sub-polar and polar regions. 
The event averaged ionization effect is maximal al altitudes 12-18 $\mathrm{km}$ a.s.l. and it is in the order of 80-95\%. The event averaged ionization effect diminishes in the low stratosphere and troposphere. The $24^{h}$ ionization effect relative to GCR is minor in a low stratosphere and troposphere and it is uniformly distributed in both hemispheres. The weekly averaged ionization effect relative to GCR is clearly negative in low stratosphere and troposphere, because of the Forbush decrease reducing the GCR flux. Therefore, at weekly time scales the GCR govern the ionization effect even during moderately strong events as was reported in Usoskin et al. (2011b).

The computed ion production rates, accordingly ionization effect at various time scales allow one to study the possible influence of SEPs on atmospheric processes over different time scales (e.g. Bazilevskaya et al., 2008; Mironova et al., 2015) and/or be used as a proxy to asses the radiation environment in the atmosphere, accordingly aircrew exposure (Vainio et al., 2009).

\section{Acknowledgements}

A. Mishev is grateful to the staff of Sodankylä Geophysical Observatory (Oulu unit), University of Oulu, Finland for the given computational time. We warmly acknowledge Prof. Ilya Usoskin from Oulu University-Finland for the discussions related to the application of cosmic ray induced ionization model. This work was supported by the Academy of Finland (project 272157, Center of Excellence ReSoLVE). We warmly acknowledge the PIs of NM stations included in NMDB, kindly providing data for this study.

\section{References}

Agostinelli, S., Allison, J., Amako, K., 2003. GEANT 4 - a simulation toolkit. Nuclear Instruments and Methods in Physics Research Section A: Accelerators, Spectrometers, Detectors and Associated Equipment 506 (3), 250-303. Aschwanden, M., 2012. GeV particle acceleration in solar flares and ground level enhancement (GLE) events. Space Science Reviews 171 (1-4), 3-21.

Bazilevskaya, G. A., 2005. Solar cosmic rays in the near earth space and the atmosphere. Advances in Space Research 35 (3), 458-464.

Bazilevskaya, G. A., Usoskin, I. G., Flückiger, E., Harrison, R., Desorgher, L., Bütikofer, B., Krainev, M., Makhmutov, V., Stozhkov, Y., Svirzhevskaya, A., Svirzhevsky, N., Kovaltsov, G., 2008. Cosmic ray induced ion production in the atmosphere. Space Science Reviews 137, 149-173. 
Bieber, J., Droge, W., Evenson, P., Pyle, K., Ruffolo, D., Pinsook, U., Tooprakai, P., Rujiwarodom, M., Khumlumlert, T., Krucker, S., 2002. Energetic particle observations during the 2000 July 14 solar event. Astrophysical Journal 567 (1), 622-634.

Bombardieri, D., Duldig, M., Michael, K., Humble, J., 2006. Relativistic proton production during the 2000 July 14 solar event: The case for multiple source mechanisms. Astrophysical Journal 644 (1), 565-574.

Burger, R., Potgieter, M., Heber, B., 2000. Rigidity dependence of cosmic ray proton latitudinal gradients measured by the ulysses spacecraft: Implication for the diffusion tensor. Journal of Geophysical Research 105, 27447-27445.

Cliver, E., Kahler, S., Reames, D., 2004. Coronal shocks and solar energetic proton events. Astrophysical Journal 605, 902-910..

Cooke, D., Humble, J., Shea, M., Smart, D., Lund, N., Rasmussen, I., Byrnak, B., Goret, P., Petrou, N., 1991. On cosmic-ray cutoff terminology. Il Nuovo Cimento C 14 (3), 213-234.

Desorgher, L., Flückiger, E., Gurtner, M., Moser, M., Bütikofer, R., 2005. A GEANT 4 code for computing the interaction of cosmic rays with the earth's atmosphere. Internationl Journal of Modern Physics A 20 (A11), 6802-6804.

Desorgher, L., Kudela, K., Flückiger, E., Bütikofer, R., Storini, M., Kalegaev, V., 2009. Comparison of Earth's magnetospheric magnetic field models in the context of cosmic ray physics. Acta Geophysica 57 (1), 75-87.

Dorman, L., 2004. Cosmic Rays in the Earth's Atmosphere and Underground. Kluwer Academic Publishers, Dordrecht.

Dorman, L., 2009. The Role of Space Weather and Cosmic Ray Effects in Climate Change. in Climate Change: Observed impacts on Planet Earth, Elsevier, Amsterdam.

Dryer, M., Fry, C., Sun, W., Deehr, C., Smith, Z., Akasofu, S.-I., Andrews, M., 2001. Prediction in real time of the 2000 July 14 heliospheric shock wave and its companions during the 'Bastille' epoch. Solar Physics 204 (1-2), $267-286$.

Forbush, S., 1937. On the effects in cosmic-ray intensity observed during the recent magnetic storm. Physical Review 51 (12), 1108-1109.

Gaisser, T. K., Stanev, T., 2010. Cosmic rays. in: Nakamura K. N. et al., Review of Particle Physics. Journal of Physics G 37, 269-275.

Gleeson, L., Axford, W., 1968. Solar modulation of galactic cosmic rays. Astrophysical Journal 154, 1011-1026.

Gopalswamy, N., Xie, H., Yashiro, S., Akiyama, S., Mäkelä, P., Usoskin, I., 2012. Properties of ground level enhancement events and the associated solar eruptions during solar cycle 23. Space Science Reviews 171 (1-4), 23-60.

Jackman, C., Marsh, D., Vitt, F., Garcia, R., Fleming, E., Labow, G., Randall, C., Lpez-Puertas, M., Funke, B., Von Clarmann, T., Stiller, G., 2008. Shortand medium-term atmospheric constituent effects of very large solar proton events. Atmospheric Chemistry and Physics 8 (3), 765-785.

Jackman, C., Marsh, D., Vitt, F., Roble, R., Randall, C., Bernath, P., Funke, 
B., Lpez-Puertas, M., Versick, S., Stiller, G., Tylka, A., Fleming, E., 2011. Northern hemisphere atmospheric influence of the solar proton events and ground level enhancement in January 2005. Atmospheric Chemistry and Physics 11 (13), 6153-6166.

Klein, K.-L., Trottet, G., Lantos, P., Delaboudinire, J.-P., 2001. Coronal electron acceleration and relativistic proton production during the 14 July 2000 flare and CME. Astronomy and Astrophysics 373 (3), 1073-1082.

Krivolutsky, A., Klyuchnikova, A., Zakharov, G., Vyushkova, T., Kuminov, A., 2006. Dynamical response of the middle atmosphere to solar proton event of July 2000: Three-dimensional model simulations. Advances in Space Research 37 (8), 1602-1613..

Krivolutsky, A., Repnev, A., 2012. Impact of space energetic particles on the Earth's atmosphere (a review). Geomagnetism and Aeronomy 52 (6), 685716.

Kudela, K., Bučik, R., Bobik, P., 2008. On transmissivity of low energy cosmic rays in disturbed magnetosphere. Advances in Space Research 42 (7), 13001306.

Kudela, K., Usoskin, I., 2004. On magnetospheric transmissivity of cosmic rays. Czechoslovak Journal of Physics 54 (2), 239-254.

Langel, R., 1987. Main field in geomagnetism. In: Geomagnetism. J.A. Jacobs Academic Press, London, Ch. 1, pp. 249-512.

Mavromichalaki, H., Papaioannou, A., Plainaki, C., Sarlanis, C., Souvatzoglou, G., Gerontidou, M., Papailiou, M., Eroshenko, E., Belov, A., Yanke, V., Flückiger, E., Bütikofer, R., Parisi, M., Storini, M., Klein, K.-L., Fuller, N., Steigies, C., Rother, O., Heber, B., Wimmer-Schweingruber, R., Kudela, K., Strharsky, I., Langer, R., Usoskin, I., Ibragimov, A., Chilingaryan, A., Hovsepyan, G., Reymers, A., Yeghikyan, A., Kryakunova, O., Dryn, E., Nikolayevskiy, N., Dorman, L., Pustil'Nik, L., 2011. Applications and usage of the real-time neutron monitor database. Advances of Space Research 47, 2210-2222.

McCracken, K., Rao, V., Shea, M., 1962. The trajectories of cosmic rays in a high degree simulation of the geomagnetic field. Technical Report 77, Massachusetts Institute of Technology, Cambridge, MA, USA.

Mironova, I., Aplin, K., Arnold, F., Bazilevskaya, G., Harrison, R., Krivolutsky, A., Nicoll, K., Rozanov, E., Turunen, E., Usoskin, I., 2015. Energetic particle influence on the Earth's atmosphere. Space Science Reviews, 96.

Mironova, I., Usoskin, I., 2014. Possible effect of strong solar energetic particle events on polar stratospheric aerosol: A summary of observational results. Environmental Research Letters 9 (1), 015002.

Mironova, I., Usoskin, I., Kovaltsov, G., Petelina, S., 2012. Possible effect of extreme solar energetic particle event of 20 January 2005 on polar stratospheric aerosols: Direct observational evidence. Atmospheric Chemistry and Physics 12 (2), 769-778.

Mishev, A., Usoskin, I., 2013. Computations of cosmic ray propagation in the earth's atmosphere, towards a GLE analysis. Journal of Physics: Conference 
Series 409, 012152.

Mishev, A., Usoskin, I., 2016. Analysis of the ground level enhancements on 14 July 2000 and on 13 December 2006 using neutron monitor data. Solar Physics 291 (4), 1225-1239.

Mishev, A., Velinov, P., 2007. Atmosphere ionization due to cosmic ray protons estimated with corsika code simulations. Comptes Rendus de L'Academie Bulgare des Sciences 60 (3), 225-230.

Mishev, A., Velinov, P., 2010. The effect of model assumptions on computations of cosmic ray induced ionization in the atmosphere. Journal of Atmospheric and Solar-Terrestrial Physics 72 (5-6), 476-481.

Mishev, A., Velinov, P., 2011a. Normalized ionization yield function for various nuclei obtained with full monte carlo simulations. Advances of Space Research 48 (1), 19-24.

Mishev, A., Velinov, P., 2011b. Renormalized ionization yield function y for different nuclei obtained with full monte carlo simulations. Comptes Rendus de L'Academie Bulgare des Sciences 64 (7), 997-1006.

Mishev, A., Velinov, P., 2013. A maverick GLE 70 in solar minimum. calculations of enhanced ionization in the atmosphere due to relativistic solar energetic particles. Comptes Rendus de L'Academie Bulgare des Sciences 66 (10), 1457-1462.

Mishev, A., Velinov, P., 2014. Influence of hadron and atmospheric models on computation of cosmic ray ionization in the atmosphere-extension to heavy nuclei. Journal of Atmospheric and Solar-Terrestrial Physics 120, 111-120.

Mishev, A., Velinov, P., 2015. Time evolution of ionization effect due to cosmic rays in terrestrial atmosphere during GLE 70. Journal of Atmospheric and Solar-Terrestrial Physics 129, 78-86.

Mishev, A., Velinov, P., 2016. Ionization effect due to cosmic rays during Bastille day event-GLE 59 on short and mid time scales. Comptes Rendus de L'Academie Bulgare des Sciences 69 (11), 1479-1484.

Mishev, A., Velinov, P., Mateev, L., Tassev, Y., 2011. Ionization effect of solar protons in the earth atmosphere - case study of the 20 january 2005 sep event. Advances of Space Research 48 (7), 1232-1237.

Mishev, A., Velinov, P., Mateev, L., Tassev, Y., 2013. Ionization effect of nuclei with solar and galactic origin in the earth atmosphere during GLE 69 on 20 January 2005. Journal of Atmospheric and Solar-Terrestrial Physics 89 (1), $1-7$.

Moraal, H., McCracken, K., 2012. The time structure of ground level enhancements in solar cycle 23. Space Science Reviews 171 (1-4), 85-95.

Nevalainen, J., Usoskin, I., Mishev, A., 2013. Eccentric dipole approximation of the geomagnetic field: Application to cosmic ray computations. Advances in Space Research 52 (1), 22-29.

Nicoll, K., Harrison, R., 2014. Detection of lower tropospheric responses to solar energetic particles at midlatitudes. Physical Review Letters 112 (22), 225001.

O'Brien, K., 1970. Calculated cosmic ray ionization in the lower atmosphere. 
Journal of Geophysical Research 75 (22), 4357-4359.

Pfotzer, G., 1936. Dreifachkoinzidenzen der ultrastrahlung aus vertikaler richtung in der stratosphäre. Zeitschrift für Physik 102 (1-2), 23-40.

Picone, J., Hedin, A., Drob, D., Aikin, A., 2002. NRLMSISE-00 empirical model of the atmosphere: Statistical comparisons and scientific issues. Journal of Geophysical Research: Space Physics 107 (A12), 1468.

Porter, H., Jackman, C., Green, A., 1976. Efficiencies for production of atomic nitrogen and oxygen by relativistic proton impact in air. The Journal of Chemical Physics 65 (1), 154-167.

Randall, C., Harvey, V., Singleton, C., Bailey, S., Bernath, P., Codrescu, M., Nakajima, H., Russell, J., 2007. Energetic particle precipitation effects on the southern hemisphere stratosphere in 1992-2005. Journal of Geophysical Research Atmospheres 112 (8), D08308.

Reames, D., 1999. Particle acceleration at the sun and in the heliosphere. Space Science Reviews 90 (3-4), 413-491.

Regener, E., Pfotzer, G., 1935. Vertical intensity of cosmic rays by threefold coincidences in the stratosphere. Nature 136, 718-719.

Rozanov, E., Calisto, M., Egorova, T., Peter, T., Schmutz, W., 2012. Influence of the precipitating energetic particles on atmospheric chemistry and climate. Surveys in Geophysics 33 (3-4), 483-501.

Shea, M., Smart, D., 1982. Possible evidence for a rigidity-dependent release of relativistic protons from the solar corona. Space Science Reviews 32, 251-271.

Shea, M., Smart, D., 1990. A summary of major solar proton events. Solar Physics 127, 297-320.

Shea, M., Smart, D., McCracken, K., 1965. A study of vertical cut off rigidities using sixth degree simulations of the geomagnetic field. Journal of Geophysical Research 70, 4117-4130.

Smart, D., Shea, M., Flückiger, E., 2000. Magnetospheric models and trajectory computations. Space Science Reviews 93 (1), 305-333.

Stoker, P., 1995. Relativistic solar proton events. Space Science Reviews 73 (34), 327-385.

Tsyganenko, N., 1989. A magnetospheric magnetic field model with a warped tail current sheet. Planetary and Space Science 37 (1), 5-20.

Usoskin, I., Bazilevskaya, G., Kovaltsov, G., 2011a. Solar modulation parameter for cosmic rays since 1936 reconstructed from ground-based neutron monitors and ionization chambers. Journal of Geophysical Research 116, A02104.

Usoskin, I., Kovaltsov, G., 2006. Cosmic ray induced ionization in the atmosphere: Full modeling and practical applications. Journal of Geophysical Research 111 (D21206).

Usoskin, I., Kovaltsov, G., Mironova, I., Tylka, A., Dietrich, W., 2011b. Ionization effect of solar particle gle events in low and middle atmosphere. Atmospheric Chemistry and Physics 11, 1979-1988.

Usoskin, I. G., Desorgher, L., Velinov, P., Storini, M., Flückiger, E., Bütikofer, 
R., Kovaltsov, G., 2009. Ionization of the earth's atmosphere by solar and galactic cosmic rays. Acta Geophysica 57 (1), 88-101.

Vainio, R., L. Desorgher, D. Heynderickx, M. Storini, E. Flückiger, et al., 2009. Dynamics of the Earth's particle radiation environment. Space Science Reviews, 147 (3-4), 187-231, 2009.

Velinov, P., Asenovski, S., Kudela, K., Lastovička, J., Mateev, L., Mishev, A., Tonev, P., 2013. Impact of cosmic rays and solar energetic particles on the earth's ionosphere and atmosphere. Journal of Space Weather and Space Climate 3, A14.

Velinov, P., Mishev, A., Mateev, L., 2009. Model for induced ionization by galactic cosmic rays in the earth atmosphere and ionosphere. Advances in Space Research 44 (9), 1002-1007.

Verronen, P., Andersson, M., Kero, A., Enell, C.-F., Wissing, J., Talaat, E., Kauristie, K., Palmroth, M., Sarris, T., Armandillo, E., 2015. Contribution of proton and electron precipitation to the observed electron concentration in October-November 2003 and September 2005. Annales Geophysicae 33 (3), 381-394.

Vitt, F., Jackman, C., 1996. A comparison of sources of odd nitrogen production from 1974 through 1993 in the earth's middle atmosphere as calculated using a two-dimensional model. Journal of Geophysical Research Atmospheres 101 (D3), 6729-6739.

Žigman, V., Kudela, K., Grubor, D., 2014. Response of the earth's lower ionosphere to the ground level enhancement event of December 13, 2006. Advances in Space Research 53 (5), 763-775.

$\mathrm{Yu}, \mathrm{F} ., \mathrm{Luo}, \mathrm{G} ., 2$ 2014. Effect of solar variations on particle formation and cloud condensation nuclei. Environmental Research Letters 9 (4), 045004. 
Table 1

Spectral and angular characteristics apparent source position location during GLE 59 on 14 July 2000 used for the computation of ion production rate

\begin{tabular}{ccccccc}
\hline Time [UT] & $J_{0}\left[\mathrm{~m}^{-2} \mathrm{~s}^{-1} s r^{-1} G V^{-1}\right]$ & $\gamma$ & $\delta \gamma$ & $\sigma^{2}\left[\mathrm{rad}^{2}\right]$ & $\Psi$ [degrees] & $\Lambda$ [degrees] \\
\hline $10: 45$ & 312000 & 4.25 & 0.8 & 1.75 & 14.32 & -8.02 \\
$11: 00$ & 290000 & 5.51 & 0.05 & 11.5 & 10.88 & -12.65 \\
$11: 30$ & 272000 & 5.52 & 0.05 & 11.8 & 14.32 & -16.04 \\
$12: 00$ & 230000 & 5.74 & 0.02 & 12.1 & 6.3 & -21.19 \\
$12: 30$ & 195200 & 5.83 & 0.03 & 12.5 & 8.59 & -23.49 \\
$13: 00$ & 193000 & 6.01 & 0.0 & 12.6 & 6.28 & -25.78 \\
$13: 30$ & 187100 & 6.15 & 0.0 & 13.1 & 6.87 & -29.22 \\
$14: 00$ & 182500 & 6.22 & 0.0 & 13.5 & 6.91 & -32.65 \\
\hline
\end{tabular}


Figure captions:

Fig.1 Rigidity spectra and pitch angle distributions of SEPs for various moments during the GLE 59 . The SEP spectra are according to Bombardieri et al. (2006); Mishev and Usoskin (2016). Time (UT) refers to the start of the corresponding interval as denoted in the legend over which the spectra are derived. The solid line in the left panel denote GCR flux. a) Rigidity spectrum of SEPs; b) Pitch angle distribution.

Fig.2 Ion production rate due to GCR and SEPs during the Bastille day GLE 59 on 14 July 2000. a) Ion production rate in the polar region with $R_{c}$ approx $1 \mathrm{GV}$; b) Ion production rate in the region with $R_{c}$ approx $2 \mathrm{GV}$.

Fig.3 Event integrated relative to the average due to GCRs ionization effect during the Bastille day GLE 5914 July 2000. a) Ionization effect averaged over the event at $20 \mathrm{~km}$ a.s.l. in the polar and sub-polar region; b) Ionization effect averaged over the event at $15 \mathrm{~km}$ a.s.l. in the polar and sub-polar region.

Fig.4 Event integrated relative to the average due to GCRs ionization effect during the Bastille day GLE 5914 July 2000. a) Ionization effect averaged over the event at $12 \mathrm{~km}$ a.s.l. in the polar and sub-polar region; b) Ionization effect averaged over the event at $8 \mathrm{~km}$ a.s.l. iin the polar and sub-polar region.

Fig.5. $24^{h}$ averaged relative to the average due to GCRs ionization effect during the Bastille day GLE 5914 July 2000. a) $24^{h}$ ionization effect at $12 \mathrm{~km}$ a.s.l .in the polar and sub-polar region; b) $24^{h}$ ionization effect at $8 \mathrm{~km}$ a.s.l. in the polar and sub-polar region.

Fig.6. Weekly averaged relative to the average due to GCRs ionization effect during the Bastille day GLE 5914 July 2000. a) Weekly ionization effect at $12 \mathrm{~km}$ a.s.l. in the polar and sub-polar region; b) Weekly ionization effect at $8 \mathrm{~km}$ a.s.l. in the polar and sub-polar region. 

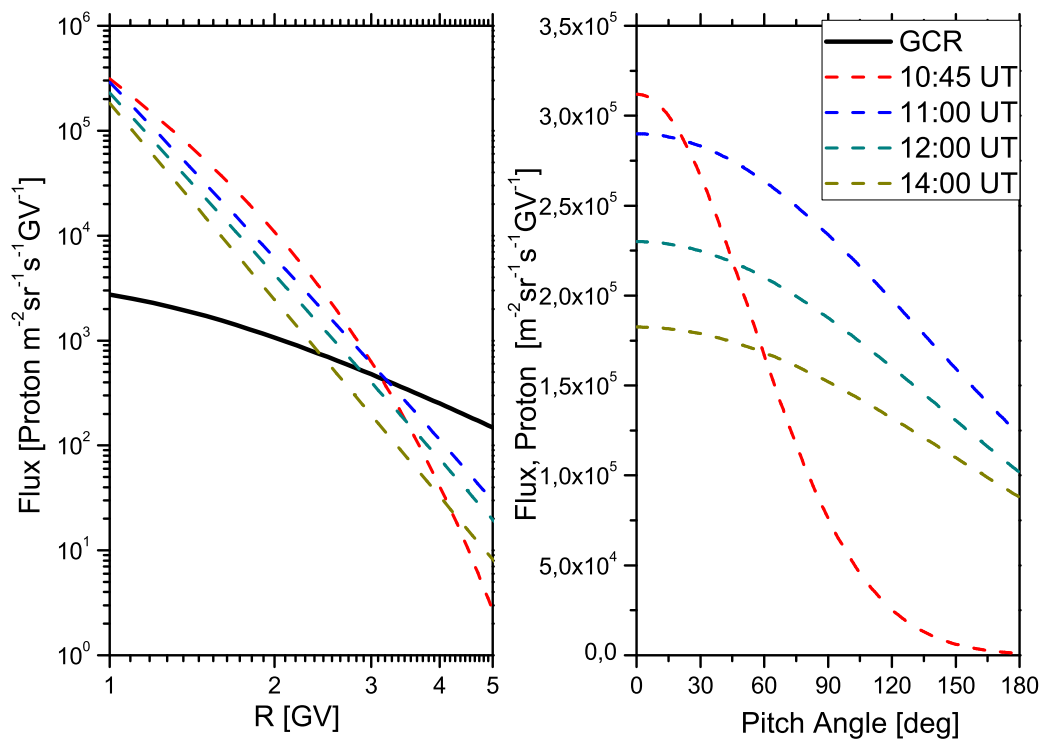

Fig. 1. Rigidity spectra and pitch angle distributions of SEPs for various moments during the GLE 59 . The SEP spectra are according to Bombardieri et al. (2006); Mishev and Usoskin (2016). Time (UT) refers to the start of the corresponding interval as denoted in the legend over which the spectra are derived. The solid line in the left panel denote GCR flux. a) Rigidity spectrum of SEPs; b) Pitch angle distribution.

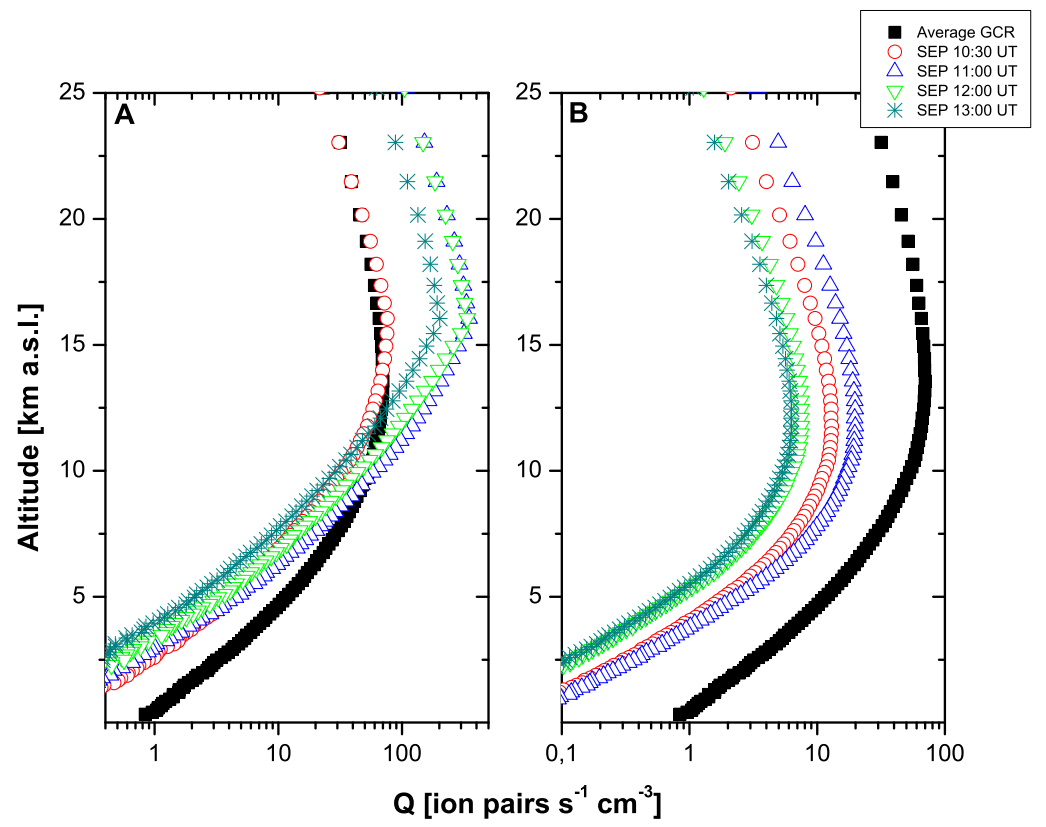

Fig. 2. Ion production rate due to GCR and SEPs during the Bastille day GLE 59 on 14 July 2000. a) Ion production rate in the polar region with $R_{c}$ approx $1 \mathrm{GV}$; b) Ion production rate in the region with $R_{c}$ approx $2 \mathrm{GV}$. 

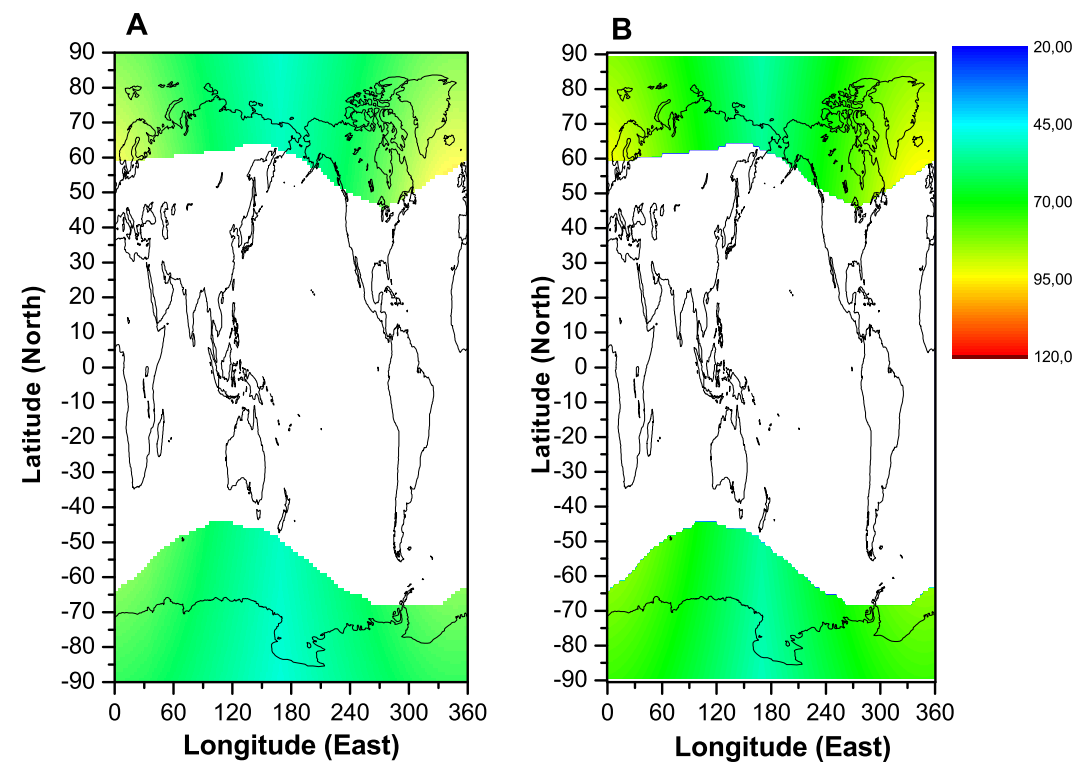

Fig. 3. Event integrated relative to the average due to GCRs ionization effect during the Bastille day GLE 5914 July 2000. a) Ionization effect averaged over the event at $20 \mathrm{~km}$ a.s.l. in the polar and sub-polar region; b) Ionization effect averaged over the event at $15 \mathrm{~km}$ a.s.l. in the polar and sub-polar region.
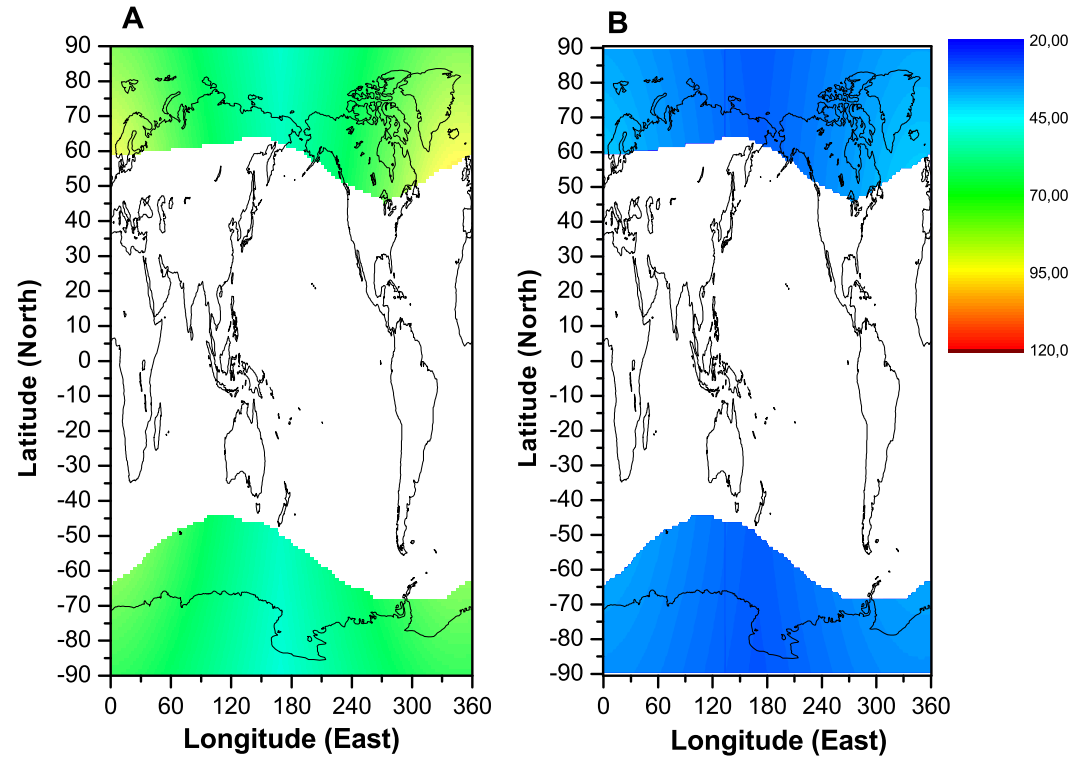

Fig. 4. Event integrated relative to the average due to GCRs ionization effect during the Bastille day GLE 5914 July 2000. a) Ionization effect averaged over the event at $12 \mathrm{~km}$ a.s.l. in the polar and sub-polar region; b) Ionization effect averaged over the event at $8 \mathrm{~km}$ a.s.l. in the polar and sub-polar region. 

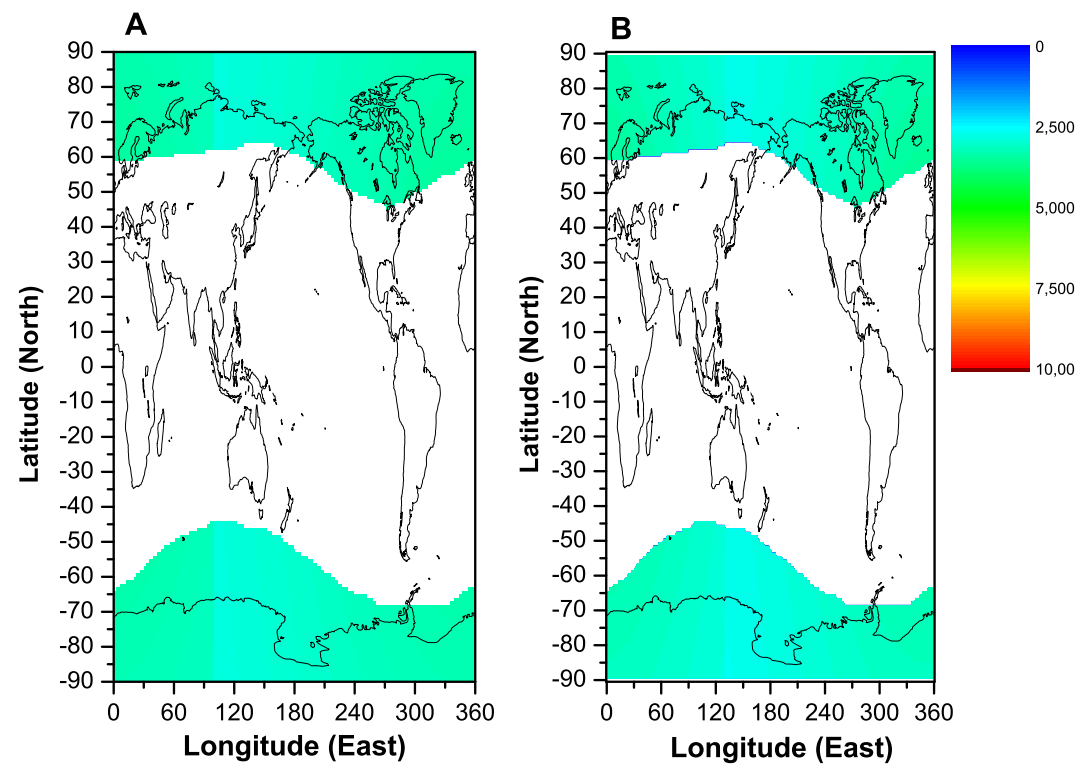

Fig. 5. $24^{h}$ averaged relative to the average due to GCRs ionization effect during the Bastille day GLE 5914 July 2000. a) $24^{h}$ ionization effect at $12 \mathrm{~km}$ a.s.l .in the polar and sub-polar region; b) $24^{h}$ ionization effect at $8 \mathrm{~km}$ a.s.l. in the polar and sub-polar region.
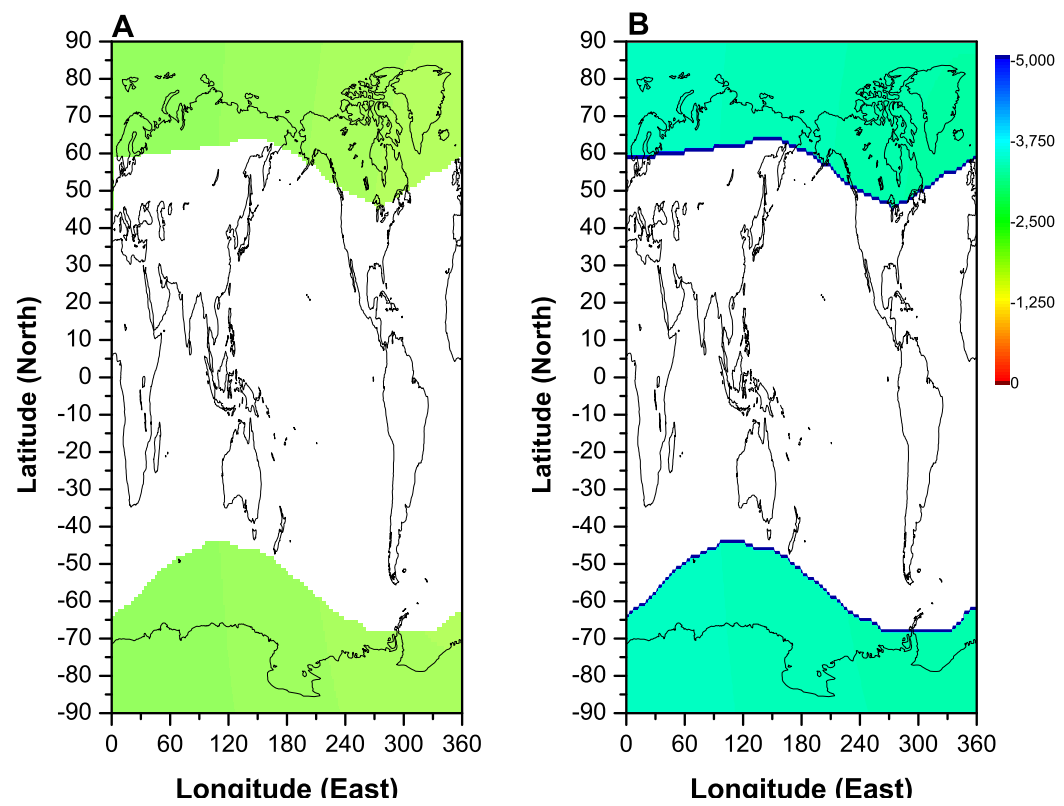

Fig. 6. Weekly averaged relative to the average due to GCRs ionization effect during the Bastille day GLE 5914 July 2000. a) Weekly ionization effect at $12 \mathrm{~km}$ a.s.l. in the polar and sub-polar region; b) Weekly ionization effect at $8 \mathrm{~km}$ a.s.l. in the polar and sub-polar region. 